\title{
Radiofrequency Mapping Prior to Dorsal Root Ganglion Stimulation in a Patient with CRPS and Transitional Vertebral ANATOMY: A CASE Report
}

\author{
Loren S. Guzman, MD, Christopher Paul, MD, Heejung Choi, MD, and Johnathan H. Goree, MD
}

Dorsal root ganglion (DRG) stimulation is an effective treatment for chronic, refractory complex regional pain syndrome (CRPS). Routinely, the placement of a DRG stimulator lead is based on established anatomical knowledge of dermatomes. In patients with lumbosacral transitional vertebral anatomy, dermatome maps are not dependable due to dermatomal variance. This can make correct placement of stimulator leads challenging as the supposed target may not actually be responsible for sensing the painful area. We present a case of a 60-year-old woman with CRPS and lumbarization of the S1 vertebral body (presence of 6 lumbar vertebrae and 4 sacral vertebrae) who failed a DRG stimulator trial after using conventional dermatome maps to identify target DRGs assuming that the nerve exiting below L6 corresponded to the S1 dermatome. Following the failed trial, sensory stimulation of the DRG via radiofrequency stimulation was used to accurately map dermatomes leading to a successful DRG stimulation implant at a DRG level that differed from expected based on traditional dermatome maps. Thus, DRG stimulation may guide decisionmaking in regard to target stimulation in patients with abnormal vertebral anatomy.

Key words: Dorsal root ganglion stimulation, dermatome mapping, radiofrequency stimulation, spinal anomaly, lumbosacral transitional vertebrae, complex regional pain syndrome, neuromodulation, dermatomal variance
Complex regional pain syndrome (CRPS) is a painful neuropathic condition that is characterized by localized pain with vasomotor, sudomotor, and motor changes. Treatment options include analgesics, tricyclic antidepressants, antiepileptic drugs, sympathetic nerve blocks, physical therapy, and neuromodulation (1).

From : Department of Anesthesiology, University of Arkansas for Medical Sciences, Little Rock, AR

Author for correspondence: Loren S. Guzman, MD

Address: Department of Anesthesiology, University of Arkansas for Medical Sciences, 4301 W. Markham Street, Slot \# 515, Little Rock, AR, 72205-7199

E-mail: Lsguzman@uams.edu

Disclaimer: There was no external funding in the preparation of this manuscript. Conflict of interest: Each author certifies that he or she, or a member of his or her immediate family, has no commercial association (i.e., consultancies, stock ownership, equity interest, patent/licensing arrangements, etc.) that might pose a conflict of interest in connection with the submitted manuscript.
Spinal cord stimulation (SCS) has been proven very effective in the use of failed back surgery syndrome and CRPS type $1(2,3)$. Serious complications occur very rarely $(4,5)$. However, SCS does have some weaknesses. Only $40 \%$ to $50 \%$ of CRPS patients achieve $>50 \%$ pain relief from SCS, and SCS also causes unpleasant paresthesias to areas outside of the painful region (6).

Since the dorsal root ganglion (DRG) is known to play a role in the development and maintenance of neuropathic pain, the DRG became a target of stimulation. DRG stimulation has several advantages compared to these SCS limitations. While SCS leads cover several dermatomes, DRG stimulation can provide pain relief to a single dermatome and offers more optimal coverage for traditionally challenging targets like focal foot pain (7). In 2017, Deer et al found that DRG stimulation was superior to SCS in the treatment of CRPS (8). 


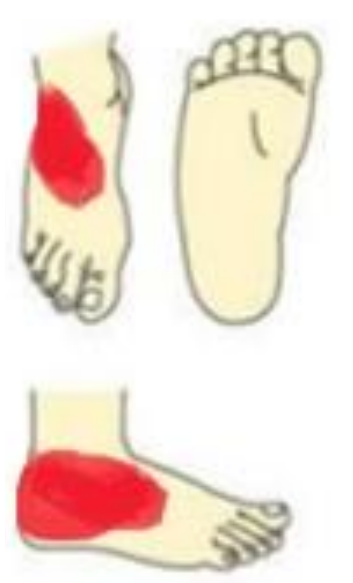

Fig. 1. Patient's painful areas.

Legend: Anterior/posterior, medial, and lateral view of patient's painful areas due to complex regional pain syndrome (CRPS). Painful areas shaded in red.

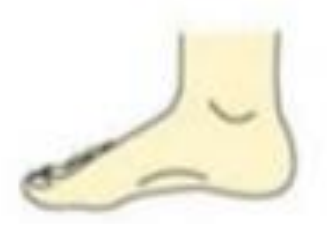

ten consent for publication of this report was given by the patient after review of the case.

\section{CASE DESCRIPTION}

This patient is a 60-year-old woman with noncontributory past medical history, social history, or family history who initially presented with 8 years of pain in the right lateral ankle, heel, and proximal dorsum of the foot that started after she had a ganglion cyst removed (Fig. 1). She underwent 3 surgical revisions, which were unsuccessful at mitigating her symptoms. She was subsequently diagnosed with CRPS of the heel and foot that failed to improve after medication therapy with gabapentin, tricyclic antidepressants, nonsteroidal anti-inflammatories, and bisphosphonates; multiple rounds of physical therapy; desensitization; and both thermal and chemical radiofrequency ablation (RFA) of peripheral nerves in her ankle and foot at an outside institution. She did have temporary relief (one week) with the first of 2 lumbar sympathetic blocks (LSB) at an outside facility. Unfortunately, the second LSB at the outside facility caused increased pain.

In 2018, a consensus committee was formed to discuss best practices for DRG stimulation. Proper placement of DRG leads for commonly treated painful areas were discussed and chosen based on dermatome maps (9). While dermatome maps are an acceptable guide, variability can be broad even in patients with normal anatomy. In patients with vertebral anomalies, dermatome maps are much less helpful.

One example of a commonly encountered vertebral anomaly is lumbosacral transitional vertebra (LSTV). LSTV is an anomaly with a prevalence ranging from $4 \%$ to $35.9 \%$ and describes the degree of fusion of $\mathrm{L} 5$ and S1. The variants can range from sacralization (fusion of the L5 vertebra to the first vertebra of the sacrum) to lumbarization (incomplete fusion of the S1 vertebra to the rest of the sacrum). The presence of a LSTV changes the number of lumbar vertebrae. With a sacralized LSTV, 4 lumbar exist, while 6 lumbar vertebral bodies exist with lumbarization (10).

In this case report, we describe a patient with refractory CRPS and LSTV who presents for DRG stimulation. The use of dermatome mapping with sensory stimulation using radiofrequency cannulae aided in the correct placement of DRG stimulation leads and dramatic improvement of her CRPS symptoms. Writ-
After evaluation, the decision was made, per institutional protocol for the management of CRPS patients, to proceed with a set of 3 lumbar sympathetic blocks immediately followed by physical therapy while the local anesthetic effect was still present. During the first LSB, fluoroscopy demonstrated the presence of a lumbarized lumbosacral transitional vertebra (LSTV) in the anteroposterior (AP) view (Fig. 2). A 30-degree oblique view was obtained and a 22-gauge 7 -inch spinal needle was inserted laterally and advanced until contact with the vertebral body of $L 3$ occurred. In a lateral view, the needle was advanced $2 \mathrm{~mm}$ beyond the anterior border and needle placement was confirmed in the AP view. Contrast medium demonstrated good vertical spread along the anterior lumbar bodies. Twenty $\mathrm{mL}$ of $0.25 \%$ bupivacaine was injected at this site. After the procedure, she proceeded directly to physical therapy. The patient reported numbness in her thigh, but not in her heel, after this LSB.

One week later, the second LSB was performed in the same manner as described above but at the L4 vertebral body. Immediately after the block, she proceeded to her physical therapy session. Over 
the course of that week, she reported $60 \%$ pain relief and improvement in strength at the ankle. The improvement was so dramatic for her that she was able to begin riding her road bike again and cycled 11 miles that week. However, the third combined LSB and physical therapy session the following week provided no additional benefit. Although the patient had some improvement, she wished to consider DRG stimulation as she desired further improvement and had failed multiple therapies previously.

She underwent DRG stimulator electrode placement without complication at the right L4 and L6 DRG. The decision was made to proceed at these levels with the assumption that the L6 DRG would be the functional S1. During this trial, she reported only $50 \%$ pain relief in her foot and heel but $80 \%$ to $90 \%$ in her leg and ankle.

Since the coverage was not ideal and she had the presence of abnormal spinal anatomy, the decision was made to undergo dermatome mapping of L5, L6, $\mathrm{S} 1$, and $\mathrm{S} 2$ nerve roots using Venom radiofrequency cannulae (Stryker, MultiGen II, Kalamazoo, MI), with a frequency of $50 \mathrm{mHz}$ and an amplitude of $1.3 \mathrm{~V}$, which is standard for radiofrequency stimulation prior to ablation for other procedures. With stimulation of L6 (S1 in normal variants), the patient reported paresthesias on the lateral ankle and S1 stimulation (S2 in normal variants) provoked paresthesias on the top and lateral border of the foot. S2 (S3 in normal variants) stimulation provided coverage of her medial heel and bottom of foot. After dermatome mapping, the patient confirmed that her pain would be $100 \%$ covered by stimulating the DRG of L6 and S1.

Based on our new dermatome map, she underwent DRG stimulator implantation with an electrode at L6-S1 and S1 on the right (Fig. 3). Immediately post procedure, the patient expressed full coverage of her painful areas. At the 6-week follow-up, the patient expressed $75 \%$ improvement of her pain.

\section{DISCUSSION}

This is the first case report of dermatomal mapping in a patient with a transitional vertebral body leading to successful identification of appropriate DRG placement, with the final targets differing from what

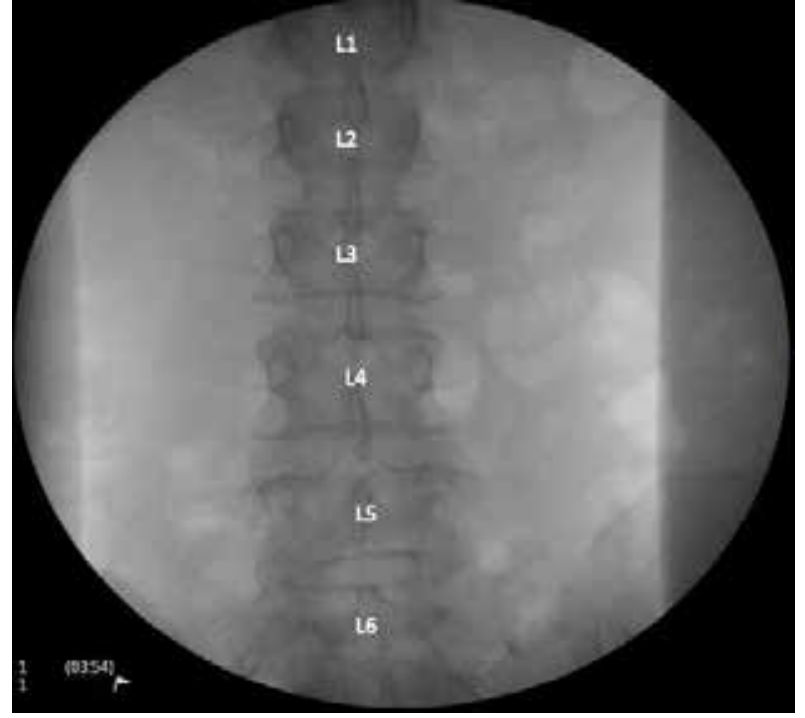

Fig. 2. Anterior/posterior fluoroscopic view of patient's lumbar spine.

Legend: Note 6 lumbar vertebrae due to lumbarization of 1st sacral segment. Vertebral bodies numbered as discussed in case report.

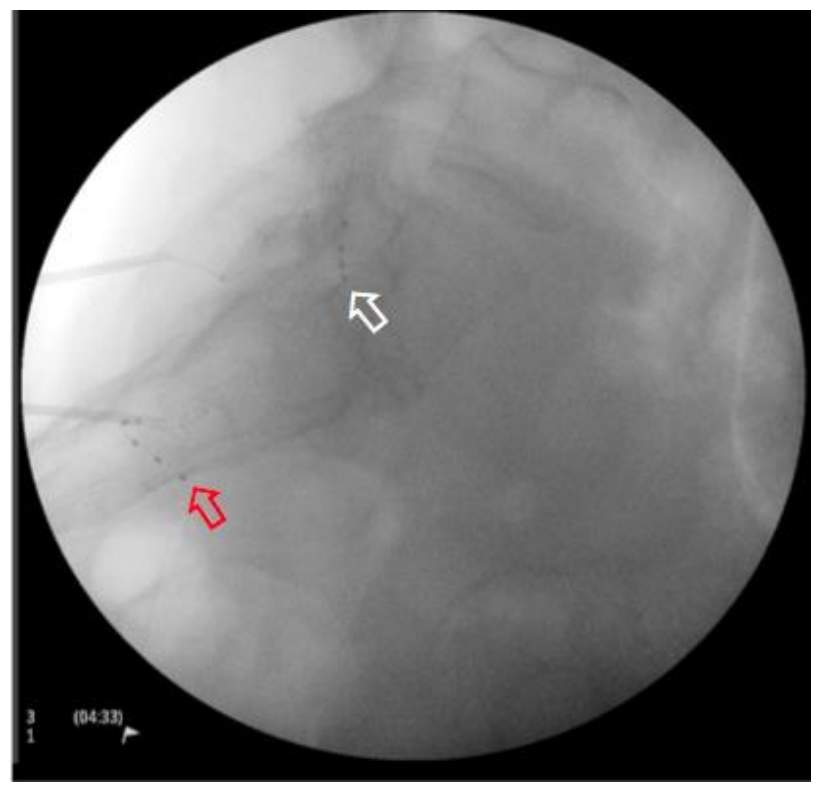

Fig. 3. Lateral fluoroscopic view of final placement of dorsal root ganglion leads.

Legend: White arrow pointing to L6-S1 lead and red arrow pointing to S1 lead. 
would be expected based on published dermatome maps. Targets for DRG stimulation are currently decided based on the dermatome in which the focus of pain lies. However, there is great variability in dermatome distribution amongst patients. There is also dramatic variability among dermatome maps. In some instances, different dermatome maps are found even in different editions of the same text (11). Several reasons can explain this discrepancy. Variability exists between individuals and is sometimes even present within an individual. Dermatomes can overlap and change size over time in the presence of lesions (12).

While all these reasons can make it difficult to determine the dermatome affected by pain, it can become even more ambiguous in the setting of spinal anomalies. McCulloch and Waddell found that the functional L5 nerve root exits at different levels in patients with LSTV. The functional L5 nerve roots exits between L6 and $\mathrm{S} 1$ in those with a lumbarized $\mathrm{S} 1$, while exiting between L4 and S1 in a patient with a sacralized L5 (13). A more recent study found that the functional nerve roots differ from anatomical nerve roots only in patients with a sacralized L5 and it did not differ in those with lumbarization (14). However, this study was small and a solid conclusion could not be drawn.

Since dermatomal variability can make it difficult to identify targets for neuromodulation with DRG, some physicians have begun dermatome mapping on patients prior to trial and implantation of DRG stimulators to improve outcomes. This was first done in 2013 by Ziudema and colleagues using retrograde transforaminal paresthesia mapping (RTPM) to deter- mine targets for DRG stimulator electrode placement for refractory groin pain. The patients in their 3-case series reported $90 \%$ to $100 \%$ pain relief after DRG stimulator implantation based on the paresthesia mapping performed preoperatively (15).

In 2017, Hunter and colleagues used radiofrequency (RF) stimulation at the DRG, similar to the technique performed in this case report, to aid in DRG lead placement for stimulator trials in patients with postamputation pain, a condition also difficult to treat and often progressive in nature. They used RF stimulation 7 to 10 days prior to trial lead implantation to determine DRG targets. The patients reported $65 \%$ to $90 \%$ pain relief after the trial period (16).

In disease processes like CRPS, DRG stimulation is often used after all other conservative treatment options have failed. The risk of failure due to improper trial placement is not acceptable due to spinal anomaly. As demonstrated in this case report, dermatomal mapping can demonstrate that the involved DRG may differ from what is expected based on dermatome maps. Thus, we feel that all patients with any suspicion of transitional anatomy or any other vertebral anomaly would benefit from dermatome mapping prior to a DRG stimulation trial. We believe that this is a safe and cost-effective way to provide a more individualized approach to neuromodulation of the DRG. While admittedly more research must be conducted to further guarantee the safety of this mapping technique, we are hopeful that wider utilization of pretrial mapping could lead to further success with DRG stimulation and decreased overall cost of treatment. 
Mapping for DRG Stimulator in Abnormal Spinal Anatomy

\section{REFERENCES}

1. Borchers AT, Gershwin ME. Complex regional pain syndrome: A comprehensive and critical review. Autoimmun Rev 2014; 13:242-265.

2. Gopal H, Fitzgerald J, McCrory C. Spinal cord stimulation for FBSS and CRPS: A review of 80 cases with on-table trial of stimulation. J Back Musculoskelet Rehabil 2016; 29:7-13.

3. Turner JA, Loeser JD, Deyo RA, Sanders SB. Spinal cord stimulation for patients with failed back surgery syndrome or complex regional pain syndrome: A systematic review of effectiveness and complications. Pain 2004; 108:137-147.

4. Eldabe S, Buchser E, Duarte RV. Complications of spinal cord stimulation and peripheral nerve stimulation techniques: A review of the literature. Pain Med 2016; 17:325-336.

5. Cameron T. Safety and efficacy of spinal cord stimulation for the treatment of chronic pain: A 20-year literature review. J Neurosurg 2004; 100:254-267.

6. Geurts JA, Smits H, Kemler, MA, Brunner F, Kessles AG, van Kleef M. Spinal cord stimulation for complex regional pain syndrome type I: A prospective cohort study with long-term follow up. Neuromodulation 2013; 15:523-529.

7. Deer TR, Grigsby E, Weiner RL, Wilcosky B, Kramer JM. A prospective study of dorsal root ganglion stimulation for the relief of chronic pain. Neuromodulation 2013; 16:67-71; discussion 7172.

8. Deer TR, Levy RM, Kramer J, et al. Dorsal root ganglion stimulation yielded higher treatment success rate for complex regional pain syndrome and causalgia at 3 and 12 months: A random- ized comparative trial. Pain 2017; 158:669-681.

9. Deer TR, Pope JE, Lamer TJ, et al. The neuromodulation appropriateness consensus committee on best practices for dorsal root ganglion stimulation. Neuromodulation 2019; 22:1-35.

10. Jancuska JM, Spivak JM, Bendo JA. A review of symptomatic lumbosacral transitional vertebrae: Bertolotti's syndrome. Int ] Spine Surg 2015; 9:42.

11. Lee MW, McPhee RW, Stringer MD. An evidence-based approach to human dermatomes. Clin Anat 2008; 21:363-373.

12. Kirk EJ, Denny-Brown D. Functional variation in dermatomes in the macaque monkey following dorsal root lesions. J Compl Neurol 1970; 139:307-320.

13. McCulloch JA, Waddell G. Variation of the lumbosacral myotomes with bony segmental anomalies. J Bone Joint Surg Br 1980; 62:475-480.

14. Kim YH, Lee PB, Lee CJ, Lee SC, Kim YC, Huh J. Dermatome variation of lumbosacral nerve roots in patients with transitional lumbosacral vertebrae. Anesth Analg 2008; 106:1279-1283.

15. Zuidema X, Breel J, Wille F. Paresthesia mapping: A practical workup for successful implantation of the dorsal root ganglion stimulator in refractory groin pain. Neuromodulation 2014; 17:665-669; discussion 669.

16. Hunter CW, Yang A, Davis T. Selective radiofrequency stimulation of the dorsal root ganglion (DRG) as a method for predicting targets for neuromodulation in patients with post amputation pain: A case series. Neuromodulation 2017; 20:708-718. 
IPM Reports 